\title{
Carcinoma escamoso oral em gato jovem
}

\author{
Squamous Cell Carcinoma Oral in Young Feline
Liliana Borges de Menezes', Marcelo Seixo de Brito e Silva², Luciano Schneider da Silva ${ }^{2}$, Carlos Eduardo Chiquetto ${ }^{2}$, Marina Pacheco Miguel ${ }^{3} \&$ Mariana Moreira Andrascho $^{4}$

\begin{abstract}
Background: Oral neoplasms are common in cats. Just the percentage of oral squamous cell carcinoma (SCC) accounts for about $70 \%$ of all feline oral tumors also it is considered highly invasive and malignant. There are reports that the flea collar increases five times the risk oral SCC development. In addition, smoke exposure is also considered a risk factor, and the animals were exposed to tobacco when makes it self-cleaning. SCC well differentiated is characterized histologically by numerous keratin pearls formation and clearly evident intercellular bridges with poor mitotic activity and nuclear pleomorphism. The median age of cats with oral SCC is about 11-13 years, but, cats as young as three years and as old as twenty-one years have been previously reported. However, it was not found reports of oral SCC in cats at age younger than one year old. The aim of this article is reports one case of oral SCC feline at age of three months old. Five days after surgical procedure the patient returned to the veterinary and the owner report that it returned to eating well.

Case: It was exanimate a three months old male mixed breed cat presenting oral volume increased and the biopsy test was suggested. However, the owner returned 3 months later when the animal showed tumor $6 \mathrm{~cm}$ in diameter, dysphagia, tongue protrusion, excessive salivation, halitosis and weight loss. It was indicated a wide surgical excision of tumoral mass. The tissue was submitted to histopathology.

Discussion: The historical, macroscopic characteristics and predisponents factors may aid in carcinoma diagnosis, however, the cytologic or histological examination of tissue confirms this diagnosis. Therefore, the microscopic evaluation revealed a malignant neoplastic proliferation of stratified squamous epithelial cells desmosomal junctions evident, few foci keratin pearl formation and presence of mononuclear inflammatory reaction perifocal. A well-differentiated squamous cell carcinoma was diagnosed by histological study. Thirty days after the surgical procedure, the animal showed a complete recovery with weight increase and eating normally. Thus, surgery proves to provide excellent quality of life maintenance for a long period of time and, although it had shown no signs of recurrence at the present moment, the literature describes that SCC long-term recurrences are common. Indeed, an important question is the quality of life of the felines with oral SCC, whereas this neoplasm led to a marked decrease in eating and drinking due to pain. Therefore, the treatment target is making the possible to maintain an excellent quality of life as long as possible. With the surgery was possible the maintenance of an excellent quality of life for a longer period. An early diagnosis could have defined the need for surgical procedure before the animal presented clinical signs characteristic, whereas represent a locally invasive neoplasm with poor response to conventional treatments models and unfavorable prognosis. Actually, increased incidences of SCC have been associated with clinical and preventive veterinary medicine improvements, which led to the prolongation of life to this animal, becoming more susceptible to senile disease, but this paper reports one case of oral SCC feline at age of three months old.
\end{abstract}

Keywords: surgery, feline, oral neoplasm, squamous cell carcinoma.

Descritores: cirurgia, felino, neoplasia oral, carcinoma de células escamosas.

${ }^{1}$ Setor de Patologia, Instituto de Patologia Tropical e Saúde Pública, Universidade Federal de Goiás (UFG), Rua 235 s/n, St Universitário, CEP 74605-050 Goiânia, GO, Brasil. ²Departamento de Medicina Veterinária, Instituto Unificado de Ensino Superior Objetivo (IUESO), Goiânia. ${ }^{3}$ Setor de Patologia Geral, UFG, Campus Jataí, Jataí, GO, Brasil. ${ }^{4}$ Escola de Veterinária, UFG, Goiânia, GO. CORRESPONDÊNCIA: L.B. Menezes [lilianab3@netscape.net - Fax +55 (62) 3233-7064]. 


\section{INTRODUÇÃO}

Neoplasmas orais são frequentes em cães e gatos, nos quais os sinais clínicos incluem: inchaço facial, coriza, salivação anormal, hemorragia oral, dificuldade de se alimentar e perda de dentes. O carcinoma de células escamosas (CCE) oral faz parte de cerca de $70 \%$ dos neoplasmas orais que atingem os felinos e é considerado extremamente invasivo e maligno [6].

Alguns autores relataram que gatos que usam coleira antipulgas possuem cinco vezes mais risco de desenvolver CCE oral que os que não usam e que a exposição ao tabaco aumenta em duas vezes este risco [1]. Gatos que vivem com fumantes podem ser expostos à mesma contaminação ambiental que seus proprietários, tanto por inalação quanto por ingestão oral durante sua auto-higienização [7].

Este neoplasma é classificado, através da diferenciação histológica, em bem diferenciado, quando apresenta numerosas pérolas de queratina e pontes intercelulares claramente evidentes com mínima atividade mitótica e pleomorfismo nuclear. Moderadamente diferenciado, quando apresenta pérolas de queratina ocasionais, pobres definições de pontes intercelulares e moderada atividade. E, pouco diferenciado, quando mostra pouca diferenciação escamosa, mas com acentuada atividade mitótica, pleomorfismo nuclear e hipercromatismo [5].

O diagnóstico desse neoplasma é mais comum em gatos entre 11 e 13 anos, animais adultos com 3 anos e, alguns autores citam ainda, em pacientes idosos com 21 anos [6]. Entretanto, não foram encontrados relatos da presença do CCE oral em gatos com idade inferior a um ano. $\mathrm{O}$ objetivo deste trabalho é relatar um caso de CCE oral em felino com três meses de idade.

\section{RELATO DE CASO}

Foi atendido um animal da espécie felina, macho, sem raça definida, com três meses de idade, apresentando aumento de volume na região oral. Após a avaliação clínica, ficou constatada a presença de um tumor de coloração esbranquiçada, com 1 a 2 $\mathrm{cm}$ de diâmetro na região dos molares inferiores, tendo sido recomendada a biópsia ao proprietário. Porém, o proprietário retornou após 3 meses, quando o animal apresentava o tumor em forma de couve-flor, com $6 \mathrm{~cm}$ de diâmetro, disfagia, protusão da língua, sialorreia, halitose e perda de peso. Devido aos sinais clínicos do paciente foi indicada uma ampla excisão cirúrgica e fragmentos do tecido foram fixados a formol ${ }^{1} 10 \%$ e processados rotineiramente para histopatologia após coloração com hematoxilina e eosina.

A remoção cirúrgica foi possível dividindo o tumor em vários fragmentos e, após sua remoção, formou-se uma grande cavidade na região submandibular, optou-se pela utilização de um dreno de penrose para drenagem da cavidade. A ferida cirúrgica foi suturada com fio de poligalactina 4-0. No pós-operatório, utilizou-se amoxicilina suspensão oral ${ }^{2}$ por 7 dias e cetoprofeno ${ }^{3}$ por 3 dias, com alimentação pastosa. Cinco dias após o procedimento cirúrgico, o animal retornou para retirada do dreno e foi relatado que o animal voltou a se alimentar bem.

\section{RESULTADOS E DISCUSSÃO}

O CCE oral tem uma variedade de apresentações e, na maioria dos casos, é notada a presença de uma massa oral pelo proprietário, entretanto, em outros casos, pode estar presente halitose, perda de peso, disfagia e pitialismo, conforme foi apresentado pelo animal relatado, quando ele já apresentava 6 meses de idade [6]. O diagnóstico de CCE oral inicia-se pelo histórico, pelas lesões macroscópicas e pela identificação dos fatores predisponentes. Entretanto, o diagnóstico definitivo só pode ser obtido através do exame histopatológico [3]. No presente caso o diagnóstico precoce, por meio da citologia ou do histopatológico, poderia ter definido a necessidade de intervenção cirúrgica antes que o animal apresentasse os sinais clínicos característicos, já que se trata de um neoplasma localmente invasivo com resposta pobre às modalidades terapêuticas convencionais $\mathrm{e}$ de prognóstico desfavorável.

A microscopia revelou proliferação neoplásica a partir da camada espinhosa da epiderme, formando cordões com infiltração dérmica de caráter sólido por células neoplásicas de formato poligonal bem diferenciadas. As células apresentaram núcleo redondo ou ovalado, com nucléolos e junções desmossômicas evidentes. Nas áreas centrais, houve presença de poucos focos de queratinização forman- 

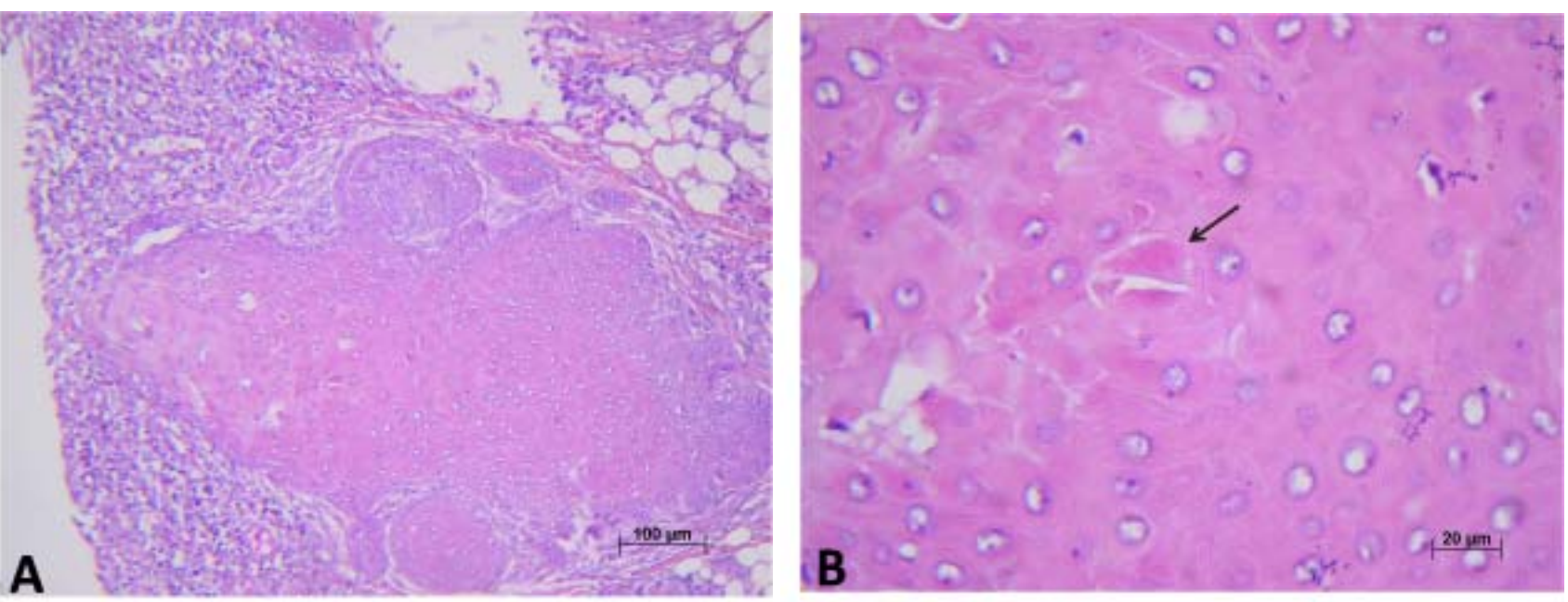

Figura 1. Fotomicrografia do carcinoma de células escamosas oral, em um gato com 6 meses. [A] Infiltração dérmica de células da camada espinhosa da epiderme (HE, 50x). [B] Focos de queratinização formando pérola córnea [seta] (HE, 400x).

do pérolas córneas, e foi observada a presença de reação inflamatória mononuclear perifocal, sendo diagnosticado carcinoma de células escamosas bem diferenciado (Figura 1), em concordância com a literatura [2].

Após 30 dias do procedimento cirúrgico, o animal apresentou restabelecimento completo com ganho de peso e alimentação normais, evidenciando que a cirurgia proporcionou a manutenção de uma excelente qualidade de vida por um período mais longo. Até o presente momento (8 meses após cirurgia), não há sinais de recidiva, entretanto, a literatura descreve que elas são frequentes a longo prazo. O diagnóstico precoce está diretamente ligado ao sucesso do tratamento, sendo que o tratamento cirúrgico deve ser realizado com ampla margem de segurança [4].
Uma outra questão importante em gatos com CCE oral é a qualidade de vida. Muitas vezes, os gatos são incapazes de se alimentar e tomar água suficientemente, devido à dor. $\mathrm{O}$ objetivo do tratamento é fazer o possível para manter uma excelente qualidade de vida pelo maior tempo possível.

De acordo com a avaliação macroscópica e histopatológica, o diagnóstico foi carcinoma escamoso oral em um gato com 6 meses de idade.

\section{NOTAS INFORMATIVAS}

${ }^{1}$ Formaldeído - Vetec Química Fina, Duque de Caxias, RJ, Brasil.

${ }^{2}$ Amoxicilina Suspensão - Eurofarma, São Paulo, SP, Brasil.

${ }^{3}$ Ketofen - Merial Saúde Animal, Campinas, SP, Brasil.

\section{REFERÊNCIAS}

1 Bertone E.R. \& Snyder L.A. 2003. Environmental and lifestyle risc factors for oral squamous cell carcinoma in domestic cats. Journal of Veterinary Medicine. 17(4): 557-562.

2 Goldschmidt M.H. \& Hendrick M.J. 2002. Tumors of the skin and soft tissues. In: Meuten J.D. (Ed.). Tumors in domestic animals. 4th. edn. Iowa: Iowa State Press, pp.145-147.

3 Guérios S., Pês M., Guimarães F., Robes R., Rodigheri S. \& Macedo T. 2003. Carcinoma de células escamosas do plano nasal em felinos: por que optar pelo tratamento cirúrgico. MEDVEP Revista Científica de Medicina Veterinária. 1(3): 203209.

4 Maffezzolli A.C. \& Zotti E.R. 2007. Carcinoma de células escamosas em felinos. 39f. Rio de Janeiro, RJ. Monografia (Pósgraduação Lato Sensu em Clínica Médica dos Felinos Domésticos) - Curso de Pós-graduação Lato Sensu em Clínica Médica dos Felinos Domésticos, Universidade Castelo Branco.

5 Maiolino P., Papparella S., Restucci B. \& De Vico G. 2001. Angiogenesis in squamous cell carcinomas of canine skin: an immunohistochemical and quantitative Analysis. Journal of Comparative Pathology. 125(2-3): 117-121. 
Menezes L. B., Brito e Silva M.S., Silva L. S., Chiquetto E., Miguel M.P. \& Andrascho M. M. 2010.Carcinoma escamoso oral em gato jovem.

6 Postorino-Reeves N.C., Turrel J.M. \& Withrow S.J. 1993. Oral squamous cell carcinoma in the cats. Journal of the American Animal Hospital Association. (29): 438-441.

7 Snyder L.A. \& Bertone E.R. 2004. p53 expression and environmental tobacco smoke exposure in feline oral squamous cell carcinoma. Veterinary Pathology. 41(3): 209-214. 